\title{
Evaluación de la condición física, práctica deportiva y estado nutricional de niños y niñas de 6 a 12 años: Estudio piloto
}

\author{
Alejandro Martínez-Rodríguez ${ }^{a, *}$, Javier Aix-Sánchez ${ }^{a}$, José Miguel Martínez-Sanz ${ }^{b, c, d}$, \\ Belén Leyva-Vela
}

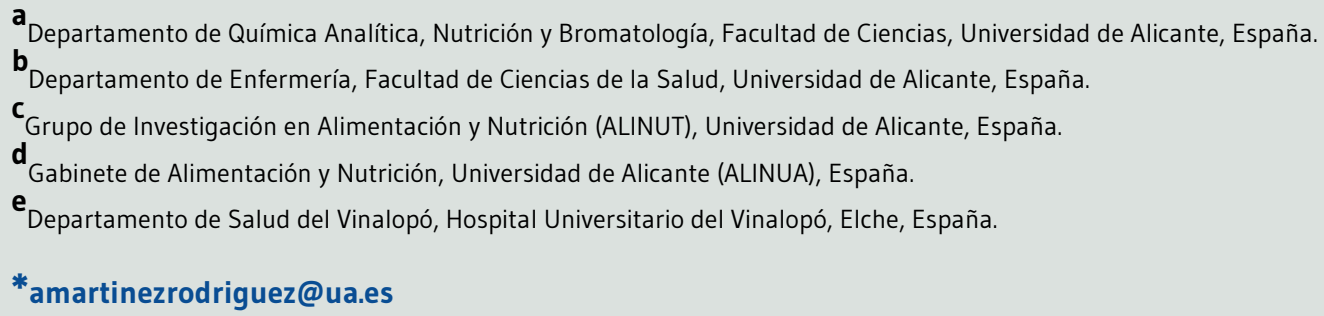

Recibido el 9 de mayo de 2016; aceptado el 19 de enero de 2017.

\section{PALABRAS CLAVE}

Sobrepeso;

Obesidad;

Escolares;

Comportamiento alimentario;

Consumo de oxígeno;

Índice de Masa

Corporal.
Evaluación de la condición física, práctica deportiva y estado nutricional de niños y niñas de 6 a 12 años: Estudio piloto

\section{RESUMEN}

Introducción: El tratamiento de la obesidad debería iniciarse dentro de la etapa escolar, centrándose en diferentes áreas de actuación, como la Educación Física. El objetivo de este estudio fue conocer el estado nutricional y el nivel de condición física en alumnos y alumnas de un centro escolar, así como la relación entre estos y la frecuencia de práctica de actividad física.

Material y Métodos: Se evaluaron 168 alumnos y alumnas entre 6-12 años del colegio concertado Azalea de Beniel (Murcia). Se registraron peso y talla (para obtener datos sobre estado nutricional), hábitos de alimentación durante el almuerzo, frecuencia de práctica de actividad física y consumo máximo de oxígeno mediante Course Navette. Se realizaron estadísticos descriptivos, comparación de medias entre los diferentes estados nutricionales y correlaciones entre estado nutricional, consumo máximo de oxígeno y frecuencia de práctica deportiva.

Resultados: Un gran número de alumnos muestran sobrepeso u obesidad. La frecuencia de práctica de actividad física registrada está por debajo de las recomendaciones de la Organización Mundial de la Salud (OMS). Se muestran correlaciones significativas entre la frecuencia de práctica de actividad física y la disminución del índice de masa corporal, así como entre el consumo máximo de oxígeno y un mayor índice de masa corporal.

Conclusiones: Una cuarta parte del alumnado presenta sobrepeso u obesidad. Persiste el consumo de productos de bollería industrial en el almuerzo. La práctica deportiva del alumnado está por debajo de las recomendaciones de la OMS. 


\section{KEYWORDS}

Overweight;

Obesity;

Child;

Feeding Behavior;

Oxygen

Consumption;

Body Mass Index.

\section{ABSTRACT}

Introduction: The adverse effects of obesity can be reversed with a good campaign to prevent obesity. This treatment should begin within the school years, focusing on different areas of activity, especially Physical Education. The aim of this study was to determine the nutritional status and level of physical fitness in students from a school, and the relationship between them and the frequency of physical activity.

Material and Methods: It was conducted in a population of 168 students between 6-12 years old in concerted Azalea Beniel School. Weight and height were recorded (for data on nutritional status), eating habits during brunch, frequency of physical activity and maximum oxygen consumption by multistage shuttle run test. We performed descriptive statistics, comparison of means, and correlations between nutritional status, maximum oxygen consumption and frequency of physical activity practice.

Results: A large number of students show nutritional status overweight or obese. The frequency of physical activity recorded is below the recommendations of the World Health Organization (WHO). Significant correlations between the frequency of physical activity and decreased body mass index are shown, as well as between the maximum oxygen consumption and increased body mass index.

Conclusions: A quarter of the students are overweight or obese. The consumption of industrial bakery products continues at lunch. The sports practice of the students is below the recommendations of the WHO.

\section{CITA}

Martínez-Rodríguez A, Aix-Sánchez J, Martínez-Sanz JM, Leyva-Vela B. Evaluación de la condición física, práctica deportiva y estado nutricional de niños y niñas de 6 a 12 años: Estudio piloto. Rev Esp Nutr Hum Diet. 2017; 21(1): 3-10. doi: 10.14306/renhyd.21.1.238

\section{INTRODUCCIÓN}

Las tendencias de obesidad y sobrepeso han sufrido un gran incremento en las últimas décadas, llegando a límites realmente alarmantes ${ }^{1}$. Según datos epidemiológicos en escolares llama la atención el alto porcentaje de obesidad infantil en España ${ }^{2}$, con un 26,2\% de niños con sobrepeso y un $18,6 \%$ de niños con obesidad en base al índice de masa corporal (IMC). Como explican Villagrán y Cols., esta situación es verdaderamente peligrosa porque en la mayoría de los casos, un niño que sufre sobrepeso llega a ser un adulto con obesidad, con todos los riesgos que esto puede acarrear ${ }^{3}$, pero no debemos olvidar que desde la infancia también se pueden sufrir una serie de trastornos relacionados con el colesterol, el aparato locomotor, la respiración, aspectos psicosociales, etc. ${ }^{4}$.
Además de sufrir sobrepeso u obesidad, España es uno de los países más sedentarios de Europa, estando las chicas en el último lugar de la lista y los chicos en posiciones no muy favorables ${ }^{5}$. Algunos de los motivos de la disminución de la práctica físico-deportiva: falta de tiempo, prefieren hacer otras cosas en su tiempo libre, pereza y desgana, influencia de los padres, estrés competitivo, poca diversión, etc. ${ }^{6}$. Como nos indica el estudio de Fernández, el tiempo final de actividad física y compromiso motor en una clase de educación física no suele superar los 15 minutos, dos o tres veces a la semana ${ }^{7}$, mientras que la recomendación de la Organización Mundial de la Salud (OMS) es que los niños y jóvenes dediquen, como mínimo, 60 minutos diarios en actividades físicas de intensidad moderada a vigorosa ${ }^{8}$. Se puede apreciar en los datos procedentes del estudio Alimentación y Valoración del Estado Nutricional de los Adolescentes (AVENA), que pone de manifiesto que la población adolescente española 
Evaluación de la condición física, práctica deportiva y estado nutricional de niños y niñas de 6 a 12 años: Estudio piloto

posee una condición física excesivamente baja en comparación con adolescentes de otros países ${ }^{9}$. Se puede encontrar relación entre los dos datos anteriores, pues un mayor riesgo de padecer sobrepeso u obesidad se asocia con un menor rendimiento en las pruebas de condición física, encontrándose relaciones inversas entre el porcentaje de masa grasa y el consumo máximo de oxígeno $\left(\mathrm{VO}_{2} \mathrm{máx}\right)$. Las relaciones encontradas entre la condición física y la composición corporal ponen de manifiesto la importancia de realizar intervenciones destinadas a mejorar la condición física, especialmente la capacidad aeróbica ${ }^{10}$. El estudio realizado por Casajús y Cols. observó que en niños y niñas con un grado superior de condición física cardiovascular, se mostraban cantidades significativamente menores de grasa subcutánea en el cuerpo entero, especialmente en la zona del tronco, concluyendo que con una mejor condición física cardiovascular se observa una composición corporal más saludable ${ }^{11}$.

Es en la edad escolar cuando a los niños se les entregan las herramientas necesarias para desenvolverse en la sociedad. La etapa de la Educación Primaria es especialmente importante, ya que en ella se inicia la escolarización obligatoria y se ponen las bases de todo el aprendizaje posterior ${ }^{12}$, por esta razón, cabe poner de manifiesto que no existe un momento más idóneo para formar a los alumnos en hábitos saludables tanto de alimentación como de realización de actividad física y mejora de sus capacidades físicas.

En este sentido, se debe tratar de educar a los alumnos y alumnas en la adquisición de hábitos saludables, pero antes, sería recomendable conocerlos profundamente, para saber los aspectos que se tendrán que corregir o modifi$\mathrm{car}^{3,13}$. Uno de los aspectos a conocer, son las elecciones alimentarias que realizan a la hora del almuerzo. El estudio de Calleja Fernández y Cols. observó que sólo el 50,3\% de los sujetos estudiados consumían algún alimento a la hora del almuerzo, también estableció la relación de que el 18,67\% de los individuos que no almorzaban presentaban sobrepeso u obesidad ${ }^{14}$. Otro estudio observó la escasa frecuencia de consumo de frutas a la hora del almuerzo ${ }^{15}$.

Por todo lo comentado anteriormente, parece evidente que existen dos factores determinantes que llevan a los escolares a una situación de sobrepeso y obesidad. Uno es la falta de ejercicio físico en la vida diaria de los niños y el otro, una alimentación inadecuada. Es por ello que se presenta la necesidad de llevar a cabo este estudio piloto, suponiendo un primer paso para futuras líneas de investigación. El objetivo de esta investigación fue describir la prevalencia de exceso de peso (sobrepeso y obesidad) y su asociación con la práctica de actividad deportiva y almuerzo en escolares de 6-12 años.

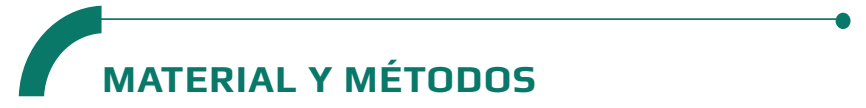

Se trata de un estudio piloto descriptivo transversal que se llevó a cabo en estudiantes escolarizados. Se estudiaron las variables: sociodemográficas, IMC, práctica de actividad deportiva, condición física y consumo de alimentos en el almuerzo.

Participaron todos los escolares ( $n=153)$, alumnos $(n=78)$ y alumnas $(n=75)$ de 6 a 12 años de un centro de Educación Primaria concertado de Beniel, población perteneciente a la Región de Murcia. El contexto socio-económico de las familias de los escolares era medio-bajo, con un $20 \%$ de familias inmigrantes. A todos los alumnos y tutores legales de éstos se les proporcionó información sobre el estudio en cuestión, y para ello, firmaron un consentimiento informado basado en la Declaración de Helsinki, para cumplir con la propuesta de principios éticos para investigación en seres humanos.

Se realizó el test Course Navette de 20 metros con etapas de un minuto, que sigue siendo el test más utilizado mundialmente, tanto en las áreas de salud, como en la escolar y deportiva. Esto se debió a la practicidad de medición, validez en un amplio rango de edades y poblaciones, fiabilidad y sensibilidad. Sin embargo, el principal motivo de su aplicabilidad se debió a la idea original de correr en un espacio reducido de $20 \mathrm{~m}$, sin la necesidad de utilizar una pista de atletismo ${ }^{16}$. Este instrumento consiste en un test en el que el sujeto se desplaza entre dos puntos, separados entre sí 20 metros, cuando se oiga la señal sonora. La velocidad aumenta de forma progresiva cada minuto y una vez que el alumno no pueda mantener el ritmo y no llegue a tiempo a alguno de los dos puntos establecidos, deberá abandonar el test. La marca obtenida en dicho test se empleará para determinar la aptitud cardiorrespiratoria y por lo tanto su $\mathrm{VO}_{2}$ máx, será el número de desplazamientos (el número de pitidos) que haya conseguido aguantar, ya que esto nos determinará su velocidad máxima alcanzada, necesaria para calcular su $\mathrm{VO}_{2}$ máx mediante la fórmula de Léger ${ }^{17}$, la cual nos indica que $\mathrm{VO}_{2}$ máx $=31,025+3,238 \mathrm{X}-3,248 \mathrm{~A}$ $+0,1536 A X$, siendo $X$ la velocidad máxima alcanzada en el test y $A$ la edad del sujeto.

Con la intención de conocer la percepción del esfuerzo del alumnado, se empleó la tabla gráfica perceptora del esfuerzo infantil. Esta herramienta está validada científicamente ${ }^{18}$, y es muy sencilla y comprensible para los alumnos y alumnas. Consiste en un gráfico en el que se representan diez niveles de esfuerzo de 1 a 10 (desde 1: "muy, muy suave" hasta 10: "tan duro que iba a parar") y que se entregó a los alumnos recién terminada la actividad para que marquen cuál es su percepción del esfuerzo en ese momento. 
Para conocer los hábitos alimentarios del alumnado en el almuerzo, se llevó a cabo una entrevista semiestructurada, de tipo retrospectivo y de los consumos regulares o más habituales de los escolares. Dicha entrevista fue realizada por un entrevistador cualificado (dietista-nutricionista).

EI IMC se calculó a partir del peso y la talla del alumnado. Para la medición del peso y talla, se siguieron las normas estandarizadas de la Sociedad Internacional para el Avance de la Cineantropometría (ISAK) ${ }^{19}$. La medición de la estatura se realizó mediante un tallímetro de pared SECA 206 (SECA, Alemania) con una precisión de $0,1 \mathrm{~cm}$; para el peso se empleó una báscula digital (Tanita, Tokio, Japón) con una precisión de 0,1kg, para posteriormente calcular su índice de masa corporal y determinar si se encontraban en bajo peso, normopeso, sobrepeso u obesidad, utilizando los criterios de la $\mathrm{OMS}^{20}$.

Para conocer la información referente a la práctica deportiva, se realizó una entrevista semiestructurada en la que los alumnos y alumnas debían de responder a preguntas sobre la frecuencia de actividad deportiva que realizaban. Esta entrevista fue Ilevada a cabo por un entrevistador cualificado (graduado en Ciencias de la Actividad física y del Deporte) en base a las indicaciones de diferentes cuestionaros sobre práctica deportiva.

El análisis estadístico se llevó a cabo a través del software SPSS ${ }^{\circledR}$ (versión 24.0, IBM). Se realizaron análisis de estadísticos descriptivos (media, desviación estándar y porcentajes), prueba de distribución de la normalidad
Kolmogorov-Smirnov para una muestra, homogeneidad de las varianzas (estadísticos de Brown-Forsythe y de Welch), comparación de medias de los distintos grupos de estudio a través del análisis de varianza (ANOVA) de un factor entresujetos y consiguientes pruebas post hoc (Tukey y GamesHowell) en función de si se cumple o no la homogeneidad de varianzas. Se emplearon pruebas paramétricas cuando los datos analizados adoptaron una distribución normal y no paramétricas cuando los datos no seguían una distribución normal. Se fijó un nivel de significación de $p<0,05$. Las asociaciones fueron verificadas con el test $\chi^{2}$ y Exacto de Fisher y las correlaciones con el test de Spearman. También se realizaron análisis de regresión múltiple.

\section{RESULTADOS}

En cuanto a las variables sociodemográficas, las edades de los sujetos que compusieron la muestra presentaron un rango de valores comprendidos entre los 6 y los 12 años cursando estudios entre $1^{\circ}$ curso y 6ำ curso de Educación Primaria. Los estadísticos descriptivos del presente estudio se muestran en la Tabla 1 , en la que se recogen los datos del consumo de alimentos durante el almuerzo e IMC en la muestra total de estudio, alumnos ( $n=78 ; 51 \%$ del total de la muestra) y alumnas ( $n=75 ; 49 \%$ del total de la muestra) de 6 a 12 años (total= 8,8 $[ \pm 1,6]$ años; alumnos $=8,7[ \pm 1,7]$ años; alumnas $=8,9[ \pm 1,5]$ años).

Tabla 1. Consumo de alimentos durante el almuerzo, IMC y frecuencia de práctica de actividad física.

\begin{tabular}{|c|c|c|c|}
\hline $\begin{array}{l}\text { Consumo de } \\
\text { alimentos en el } \\
\text { almuerzo }\end{array}$ & Población total (\%) & Alumnas (\%) & Alumnos (\%) \\
\hline Fruta & 18,4 & 23,9 & 13,5 \\
\hline Bocadillo & 41,8 & 34,3 & 48,6 \\
\hline Bollería & 10,6 & 11,9 & 9,5 \\
\hline Fruta y bocadillo & 12,8 & 10,4 & 14,9 \\
\hline Fruta y bollería & 9,9 & 14,9 & 5,4 \\
\hline Bocadillo y bollería & 6,4 & 4,5 & 8,1 \\
\hline \multicolumn{4}{|l|}{ IMC } \\
\hline Delgadez & 2,0 & 0,0 & 3,9 \\
\hline Normopeso & 72,6 & 68,0 & 76,9 \\
\hline Sobrepeso & 13,1 & 17,3 & 9,0 \\
\hline Obesidad & 12,4 & 14,7 & 10,3 \\
\hline
\end{tabular}


Evaluación de la condición física, práctica deportiva y estado nutricional de niños y niñas de 6 a 12 años: Estudio piloto

Dentro de los resultados obtenidos del IMC (total $=18,8$ $[ \pm 3,8] \mathrm{kg} / \mathrm{m}^{2} ;$ alumnos $=17,8[ \pm 3,2] \mathrm{kg} / \mathrm{m}^{2}$; alumnas $=19,9$ $[ \pm 4,0] \mathrm{kg} / \mathrm{m}^{2}$ ), cabe destacar que dentro de la población total, la prevalencia de sobrepeso fue de un $13,1 \%$ y de obesidad un $12,4 \%$, obteniéndose un total de un $25,6 \%$ de alumnos con un estado nutricional de sobrepeso-obesidad (Tabla 1). Los hábitos alimentarios asociados al almuerzo o comida de media mañana que presentan los alumnos en relación al consumo de bollería es superior al $10 \%$ en general, porcentaje que se ve ligeramente incrementado en el caso de las alumnas. El consumo de bocadillos es el que presenta resultados más elevados, sobretodo en el caso de los varones, que se sitúa en un $48,6 \%$, casi un $15 \%$ por encima del porcentaje de alumnas que consume este tipo de alimento combinado.

Los resultados del número de horas semanales de práctica deportiva, sitúan al conjunto de los escolares (alumnos y alumnas) en una media de $2,0( \pm 0,2)$ horas. Los alumnos presentan un mayor número de horas de práctica deportiva semanales $(3,7[ \pm 2,0]$ horas) frente a las alumnas $(1,9[ \pm 1,5]$ horas).
Se llevó a cabo un análisis de la posible influencia de los factores sociodemográficos en los escolares en relación al IMC, consumo de alimentos en el almuerzo, práctica deportiva y condición física. Para ello, las tablas de contingencia mostraron que existía una relación entre ser inmigrante o no con el IMC (Coeficiente de contingencia=0,713; $\left.\chi^{2}<0,001\right)$ y la frecuencia de práctica deportiva a la semana (Coeficiente de contingencia=0,520; $\left.\chi^{2}=0,019\right)$. En cuanto al IMC, 5 de los escolares inmigrantes ( $3 \%$ del total de la muestra) presentaban sobrepeso, frente a los 35 escolares no inmigrantes que presentaban sobrepeso-obesidad ( $21 \%$ del total de la muestra). Asimismo, algo similar sucedió con la frecuencia de práctica deportiva, en la que 5 de los escolares inmigrantes (3\% del total de la muestra) no realizaba ninguna práctica deportiva, frente a los 30 escolares no inmigrantes $(20 \%$ del total de la muestra) que refieren ausencia de práctica deportiva semanal. No se obtuvieron relaciones en los resultados de condición física o tipo de almuerzo entre escolares inmigrantes o no inmigrantes.

La Tabla 2 muestra la relación de práctica de actividad deportiva y condición física con el IMC de la población

Tabla 2. Relación del IMC con la condición física y práctica de actividad física semanal según sexo y en el total de la muestra.

\begin{tabular}{|c|c|c|c|}
\hline & & $\mathrm{Vo}_{2} \max$ & $\begin{array}{l}\text { N.@ de horas semanales } \\
\text { de actividad física }\end{array}$ \\
\hline POBLACIÓN TOTAL & $n=153$ & Media (Desviación típica) & Media (Desviación típica) \\
\hline Delgadez & 3 & $54,2\left( \pm 6,0 * 0^{*} * 0\right.$ & $4,33( \pm 2,1)$ \\
\hline Normopeso & 111 & $48,1( \pm 5,2)^{*}{ }^{* * 0}$ & $3,21( \pm 2,0)^{* 5 * 0}$ \\
\hline Sobrepeso & 20 & $43,9( \pm 3,8)^{* D * N}$ & $2( \pm 1,1)^{* \mathrm{~N}}$ \\
\hline Obesidad & 19 & $43,2( \pm 2,7)^{* D^{* *} \mathrm{~N}}$ & $1,32( \pm 1,5)^{* * N}$ \\
\hline ALUMNAS & n & Media (Desviación típica) & Media (Desviación típica) \\
\hline Delgadez & 0 & - & - \\
\hline Normopeso & 51 & $46,1( \pm 4,7)^{* 5 * 0}$ & $2,27( \pm 1,7)^{* * 0}$ \\
\hline Sobrepeso & 13 & $42,9( \pm 3,8)^{* \mathrm{~N}}$ & $1,77( \pm 0,7)^{* 0}$ \\
\hline Obesidad & 11 & $42,25( \pm 1,9)^{* N}$ & $0,64( \pm 0,8)^{* * N}{ }^{* S}$ \\
\hline ALUMNOS & $\mathbf{n}$ & Media (Desviación típica) & Media (Desviación típica) \\
\hline Delgadez & 3 & $54,2( \pm 6,0)^{* 0}$ & $4,33( \pm 2,1)$ \\
\hline Normopeso & 60 & $49,8( \pm 5,1)^{*}$ & 4,00 (士1,9) \\
\hline Sobrepeso & 7 & $45,7( \pm 3,2)$ & $2,43( \pm 1,6)$ \\
\hline Obesidad & 8 & $44,6( \pm 3,2)^{*} D^{* N}$ & $2,25( \pm 1,7)$ \\
\hline
\end{tabular}

* La diferencia de medias es significativa al nivel 0,05. ** La diferencia de medias es significativa al nivel 0,01.

$\mathbf{V O}_{2}$ max: consumo máximo de oxígeno; $\mathbf{D}$ : delgadez; $\mathbf{N}$ : normopeso; S: sobrepeso; O: obesidad.

Las letras iniciales indican que el grupo en cuestión tiene diferencias significativas con respecto al grupo con la letra en superíndice. 
Evaluación de la condición física, práctica deportiva y estado nutricional de niños y niñas de 6 a 12 años: Estudio piloto

estudiada. En la población total (alumnos y alumnas), se observaron diferencias significativas en el $\mathrm{VO}_{2}$ máx en función del IMC de los escolares. Aquellos escolares con delgadez o peso normal mostraron mejores resultados significativamente en $\mathrm{VO}_{2}$ máx frente a los escolares con sobrepeso u obesidad. En el caso de las alumnas, se obtuvieron diferencias significativas entre aquellas alumnas con un peso normal en comparación con las que presentaban sobrepeso u obesidad, que mostraron peores resultados en el $\mathrm{VO}_{2}$ máx. Los alumnos reportaron diferencias significativas entre los que estaban en un estado de delgadez o peso normal, con resultados incrementados en el $\mathrm{VO}_{2}$ máx, en comparación con los alumnos con obesidad.

Asimismo, en la Tabla 2 también se pueden contemplar las diferencias entre el total de la población estudiada. Los escolares con un IMC de normopeso obtuvieron diferencias significativas respecto a aquellos con sobrepeso u obesidad en el número de horas semanales de actividad deportiva, siendo los primeros los que más horas de práctica realizan. Entre las alumnas también se obtuvieron diferencias significativas en cuanto a las horas semanales de práctica deportiva. Estas diferencias se presentaron en aquellas alumnas con obesidad, cuyas horas de prácticas eran significativamente menores a las alumnas con sobrepeso o normopeso. Los alumnos no presentaron diferencias en el número de horas de práctica de actividad deportiva cuando se compararon los grupos en función del IMC.

En el presente estudio se realizaron correlaciones con la intención de conocer el grado de relación entre las variables estudiadas. Estas se llevaron a cabo en la muestra total (alumnos y alumnas), alumnos y alumnas. Las correlaciones dentro del grupo de escolares mostraron que el $\mathrm{VO}_{2}$ máx correlaciona con los días semanales de práctica de actividad deportiva $(R=0,774 ; p<0,001)$. EI IMC, también muestra correlacionarse, en este caso, negativamente con el $\mathrm{VO}_{2}$ máx $(R=-0,582 ; p<0,001)$ y con los días semanales de práctica de actividad deportiva $(R=-0,480 ; p<0,001)$. Cuando se separaron a los escolares entre alumnos y alumnas, se obtuvieron las mismas correlaciones que cuando se analizó a la muestra completa, aunque los valores fueron diferentes en alumnos $\left(\mathrm{VO}_{2}\right.$ máx y días semanales de práctica deportiva: $\mathrm{R}=0,747 ; \mathrm{p}<0,001 ; \mathrm{IMC}$ y VO $\mathrm{VO}_{2}$ máx: $\mathrm{R}=-0,528 ; \mathrm{p}<0,001$; IMC y días semanales de práctica deportiva: $R=-0,391 ; p<0,001)$ y alumnas $\left(\mathrm{VO}_{2}\right.$ máx y días semanales de práctica deportiva: $R=0,703 ; p<0,001 ; I M C$ y VO${ }_{2}$ máx: $R=-0,561 ; p<0,001$; IMC y días semanales de práctica de actividad deportiva: $R=-0,466 ; p<0,001)$.

Por último, se llevó a cabo un análisis de regresión lineal múltiple. En este sentido, el modelo de regresión lineal múltiple que tiene en cuenta al $\mathrm{VO}_{2}$ máx como variable dependiente y a los días de práctica deportiva y al IMC como predictores, presenta resultados que afirman el cumplimiento del modelo $\left(R=0,810 ; R^{2}=0,657 ; p=0,001 ;\right.$ Durbin-Watson=1,713).

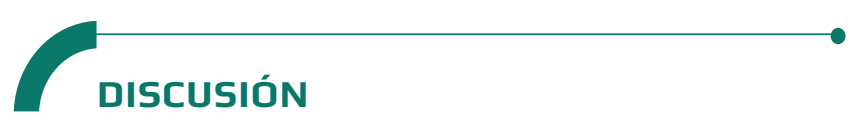

Los resultados del presente estudio indican que los niveles de sobrepeso y obesidad presentes en el centro están por debajo de la media según el estudio de ALADINO² llevado a cabo en niños y niñas españoles, con edades comprendidas entre los 6 y 9 años, en el que situaba el porcentaje de niños con sobrepeso en un $26 \%$ y de obesidad en $18,6 \%$, mientras que los resultados de este estudio nos indican un $13,1 \%$ de sobrepeso y un $12,4 \%$ de obesidad. Separando los resultados por sexo, se puede apreciar una tasa del $17,3 \%$ de sobrepeso y del $14,7 \%$ de obesidad en alumnas, porcentajes bastante similares a los del estudio comentado anteriormente, mientras que los porcentajes correspondientes a los alumnos descienden a un $9 \%$ de sobrepeso y un $10 \%$ de obesidad. En este sentido, la media del IMC de todos los alumnos de este estudio está en consonancia con la media obtenida en el estudio de ALADINO2, ya que prácticamente no hay diferencias $\left(18,8[ \pm 3,8] \mathrm{kg} / \mathrm{m}^{2}\right.$ frente a $\left.18,9[ \pm 3,8] \mathrm{kg} / \mathrm{m}^{2}\right)$. En la división de los grupos por sexo, los alumnos tuvieron un IMC similar al estudio ALADINO $\left(17,8[ \pm 3,2] \mathrm{kg} / \mathrm{m}^{2}\right.$ frente a $18,0[ \pm 2,9] \mathrm{kg} /$ $\mathrm{m}^{2}$ ). Sin embargo, en el caso de las alumnas, los resultados de este estudio están por encima de los valores promedio de las alumnas en el estudio ALADINO, así como por encima de la media de los alumnos $\left(19,9[ \pm 4,0] \mathrm{kg} / \mathrm{m}^{2}\right.$ frente a 17,8 $[ \pm 2,9] \mathrm{kg} / \mathrm{m}^{2}$ ).

Algunos motivos por los que los alumnos podrían seleccionar inadecuadamente sus almuerzos, según el estudio realizado por Macias y Cols. ${ }^{21}$, pueden ser las nuevas dinámicas familiares y la gran exposición de los niños a los comerciales alimentarios, siendo estos los causantes de la modificación de los patrones de consumo de los niños, aumentando la obesidad infantil. En el presente estudio se trató de conocer si las diferencias socioeconómicas y culturales podrían ver influida esta selección, pero los resultados obtenidos, indican que no hay una peor selección de alimentos en el almuerzo. Al respecto de la incorrecta selección de alimentos, es muy importante recuperar la dieta mediterránea, por su calidad nutricional, ya que se ha demostrado que los niveles más altos de la adherencia a un patrón de dieta mediterránea se asocia a un menor riesgo de ingestas inadecuadas ${ }^{22}$, por lo que se debe de promover este tipo de estrategia nutricional, especialmente con los grupos más vulnerables. Sería recomendable inculcar unas pautas de alimentación basadas en el consumo de alimentos de origen vegetal, donde 
Evaluación de la condición física, práctica deportiva y estado nutricional de niños y niñas de 6 a 12 años: Estudio piloto

las verduras, frutas, cereales integrales, legumbres y frutos secos son los principales alimentos que se deben consumir ${ }^{3}$. Éstas, también incluyen el consumo moderado de pescado, carnes blancas, huevos y productos lácteos. Sin embargo, el consumo de carne roja, carne procesada y los alimentos ricos en azúcares y en grasas sólo se recomiendan en pequeñas dosis y con poca frecuencia ${ }^{22}$. La adopción de esta dieta no es tarea fácil, ya que la industrialización y comercialización de la cadena alimentaria en los países occidentales y el aumento de la producción de alimentos procesados están dando lugar a una serie de cambios en la alimentación y preferencias en amplios sectores de la población ${ }^{23}$.

Es una minoría el número de alumnos que cumplen las recomendaciones mínimas de realización de actividad física que propone la $\mathrm{OMS}^{8}$. Sólo un $2 \%$ de los alumnos frente a un $0 \%$ de las alumnas, realizan 60 minutos de actividad física diaria. Debido a la asociación obtenida en este trabajo sobre la influencia de las horas de práctica sobre el nivel de condición física, se expresa la necesidad de incrementar estas horas de práctica para obtener mejoras para la salud ${ }^{24}$. En este sentido, el desarrollo de las cualidades físicas, más comúnmente conocido como condición física, ha tomado un gran valor en la sociedad actual y se relaciona con la salud y la calidad de vida. Al igual que en otros estudios, los escolares con una mejor condición física a nivel cardiovascular ${ }^{25}$, presentan menores valores de IMC, como sucede en el presente estudio. Asimismo, y en relación al resultado del análisis de regresión, los resultados obtenidos del presente estudio, destacar que concuerdan con los realizados en los estudios de Arriscado y Cols. ${ }^{10}$, así como con los de Casajús ${ }^{11}$, en los que se indica que existe una relación inversa entre el índice de masa corporal y la frecuencia de actividad física semanal, así como con su $\mathrm{VO}_{2}$ máx. Por lo tanto, el IMC y las horas de estudio van a predecir el nivel de condición física de los escolares.

No obstante, parece ser que la percepción de los progenitores del estado nutricional de sus hijos no provoca alteraciones en los hábitos de éstos sobre actividad física, disminución del sedentarismo o mejora en los hábitos alimentarios ${ }^{13}$. Es por eso que se podría destacar la influencia de los progenitores sobre la alimentación y hábitos de práctica deportiva de sus hijos como medida de control del estado nutricional de estos últimos. En este sentido, destacar que otras investigaciones ya han puesto de manifiesto la importancia de un control del IMC sobre el riesgo de presentar patologías asociadas 26,27 . Para ello, es necesario tratar de concienciar dentro del ámbito educativo, tanto familiar como escolar, la adopción de un estilo de vida saludable en el que estén presentes una buena alimentación y la realización de actividad física cotidiana, con la intención de reducir lo antes posible la prevalencia de sobrepeso y obesidad ${ }^{28,29}$.
Entre las limitaciones, comentar, en primer lugar, que esta investigación se trata de un estudio piloto con un número reducido de escolares, ya que en un futuro se pretende abordar todos los centros de la comunidad autónoma. Otra de las limitaciones del estudio es la ausencia de una mayor recogida de datos relacionados con los hábitos alimentarios mediante cuestionarios validados para ello, que complementarían la entrevista realizada. Del mismo modo ocurre con la práctica deportiva, se tendrá en cuenta la aplicación de cuestionarios específicos, con el objetivo de detallar en mayor medida las prácticas deportivas de los escolares, así como a ser posible su duración, o tiempo de compromiso motor e intensidad. En relación a la composición corporal y con vistas a futuros trabajos, los autores contemplan la posibilidad de realizar otras mediciones antropométricas como perímetro de cintura, perímetro de cadera, pliegues cutáneos, etc. Por último, también se considerará, una vez que se proceda a aumentar la muestra de estudio, presentar los datos separados por rangos de edad, ya que algunos autores mencionan que esto podría llegar a ser un factor de confusión importante.

\section{CONCLUSIONES}

El presente estudio piloto indica que una cuarta parte del alumnado presenta sobrepeso u obesidad. Asimismo, persiste el consumo de productos de bollería industrial en el almuerzo. La práctica deportiva del alumnado está, en la mayoría de los casos, por debajo de las recomendaciones de la OMS. Por último, poner de manifiesto que el número de horas semanales de práctica deportiva y el IMC pueden predecir el estado de condición física de los escolares.

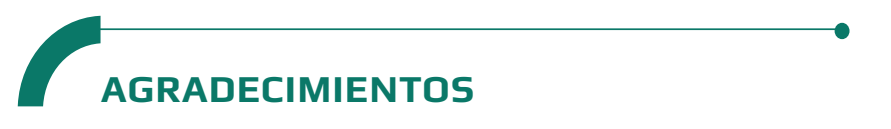

Agradecer a los alumnos, profesorado y dirección del centro de educación primaria, por su consentimiento en participar en este estudio.

\section{CONFLICTO DE INTERESES}

Los autores expresan que no hay conflictos de interés al redactar el manuscrito. 


\section{REFERENCIAS}

(1) NCD Risk Factor Collaboration (NCD-RisC). Trends in adult body-mass index in 200 countries from 1975 to 2014: a pooled analysis of 1698 population-based measurement studies with 19.2 million participants. Lancet. 2016; 387(10026): 1377-96.

(2) Agencia Española de Consumo, Seguridad Alimentaria y Nutrición. Estudio ALADINO 2013: Estudio de Vigilancia del Crecimiento, Alimentación, Actividad Física, Desarrollo Infantil y Obesidad en España 2013. Madrid: Ministerio de Sanidad, Servicios Sociales e Igualdad; 2014.

(3) Villagrán S, Rodríguez-Martín A, Novalbos JP, Martínez JM, Lechuga JL. Hábitos y estilos de vida modificables en niños con sobrepeso y obesidad. Nutr Hosp. 2010; 25(5): 823-31.

(4) Muros JJ, Som A, López H, Zabala M. Asociaciones entre el IMC, la realización de actividad física y la calidad de vida en adolescentes. (Relationships among BMI, physical activity practice and quality of life in teenagers). CCD. 2009; 4(12): 159-65.

(5) Duelo M, Escribano E, Muñoz F. Obesidad. Rev Pediatr Aten Primaria. 2009; 11(Supl. 16): S239-57.

(6) Macarro J, Romero C, Torres J. Motivos de abandono de la práctica de actividad físico-deportiva en los estudiantes de Bachillerato de la provincia de Granada. Rev Educ. 2010; 70(353): 495-519.

(7) Fernández $\mathrm{AB}$. El tiempo en la clase de educación física: la competencia docente tiempo. Deporte Act Fís. 2008; (4): 102-19.

(8) Organización Mundial de la Salud. Recomendaciones mundiales sobre actividad física para la salud. Ginebra, Suiza: Organización Mundial de la Salud; 2010.

(9) Ortega FB, Ruiz JR, Castillo MJ, Moreno LA, González-Gross M, Wärnberg ], et al. Bajo nivel de forma física en los adolescentes españoles. Importancia para la salud cardiovascular futura (Estudio AVENA). Rev Esp Cardiol. 2005; 58(08): 898-909.

(10) Arriscado D, Muros J], Zabala M, Dalmau JM. Relación entre condición física y composición corporal en escolares de primaria del norte de España (Logroño). Nutr Hosp. 2014; 30(2): 385-94.

(11) Casajús JA, Leiva MT, Ferrando JA, Moreno L, Aragonés MT, Ara I. Relación entre la condición física cardiovascular y la distribución de grasa en niños y adolescentes. Apunts Med Esport. 2006; 41(149): 7-14.

(12) Carrasco C, Alarcón R, Trianes MV. Eficacia de una intervención psicoeducativa basada en clima social, violencia percibida y sociométricos en alumnado de educación primaria. Rev Psicodidact. 2015; 20(2): 247-62.

(13) Rodríguez A, Novalbos JP, Villagran S, Martínez JM, Lechuga JL. La percepción del sobrepeso y la obesidad infantil por parte de los progenitores. Rev Esp Salud Publica. 2012; 86(5): 483-94.

(14) Calleja A, Muñoz C, Ballesteros MD, Vidal A, López J], Cano I, et al. Modificación de los hábitos alimentarios del almuerzo en una población escolar. Nutr Hosp. 2011; 26(3): 560-5.

(15) Alba E, Gandía A, Olaso G, Vallada E, Garzón MF. Consumo de frutas, verduras y hortalizas en un grupo de niños valencianos de edad escolar. Nutr clín diet hosp. 2012; 32(3): 64-71.

(16) García GC, Secchi JD. Test course navette de 20 metros con etapas de un minuto. Una idea original que perdura hace 30 años. Apunts Med Esport. 2014; 49(183): 93-103.

(17) Corral JA, del Catillo Ó. La valoración del VO2 máx. y su relación con el riesgo cardiovascular como medio de enseñanzaaprendizaje. Cuad Psicol Deporte. 2010; 10(2): 25-30.

(18) Yelling M, Lamb KL, Swaine IL. Validity of a Pictorial Perceived Exertion Scale for Effort Estimation and Effort Production During Stepping Exercise in Adolescent Children. Eur Phys Educ Rev. 2002; 8(2): 157-75.

(19) Stewart A, Marfell-Jones M. International Standards for Anthropometric Assessment. ISAK, editor. Potchefstroom, Sudáfrica: International Society for the Advancement of Kinanthropometry; 2006.

(20) WHO Multicentre Growth Reference Study Group. WHO Child Growth Standards based on length/height, weight and age. Acta Paediatr Suppl. 2006; 450: 76-85.

(21) Macias Al, Gordillo LG, Camacho EJ. Hábitos alimentarios de niños en edad escolar y el papel de la educación para la salud. Rev Chil Nutr. 2012; 39(3): 40-3.

(22) Castro-Quezada I, Román-Viñas B, Serra-Majem L. The Mediterranean diet and nutritional adequacy: a review. Nutrients. 2014; 6(1): 231-48.

(23) Durá-Gúrpide B, Durá-Travé T. Análisis nutricional del modelo dietético en alumnos de educación primaria con estado nutricional normal. Nutr Hosp. 2014; 29(6): 1311-9.

(24) Eisenmann JC, Wickel EE, Welk GJ, Blair SN. Relationship between adolescent fitness and fatness and cardiovascular disease risk factors in adulthood: the Aerobics Center Longitudinal Study (ACLS). Am Heart J. 2005; 149(1): 46-53.

(25) Mayorga-Vega D, Merino-Marban R, Rodríguez-Fernández E. Relación entre la capacidad cardiorrespiratoria y el rendimiento en los tests de condición física relacionada con la salud incluidos en la batería ALPHA en niños de 10-12 años. CCD. 2013; 8(22): 41-7.

(26) Grant-Guimaraes J, Feinstein R, Laber E, Kosoy J. Childhood Overweight and Obesity. Gastroenterol Clin North Am. 2016; 45(4): 715-28.

(27) Almerich-Torres T, Montiel-Company JM, Bellot-Arcís C, Almerich-Silla JM. Relationship between caries, body mass index and social class in Spanish children. Gac Sanit. 2016.

(28) André AL, Padez C, Rosado-Marques V, Griffiths PL, Varela-Silva MI. Growing up in Portugal: Cape Verdean ancestry children exhibit low overweight and obesity compared with Portuguese in urban Lisbon. J Biosoc Sci. 2016; 1-16.

(29) Durá-Travé T, Gallinas-Victoriano F. Dietary pattern among schoolchildren with normal nutritional status in Navarre, Spain. Nutrients. 2014; 6(4): 1475-87. 